\title{
BMP-7 suppresses excessive scar formation by activating the BMP-7/Smad1/5/8 signaling pathway
}

\author{
JINGDONG GUO, QUAN LIN, YING SHAO, LI RONG and DUO ZHANG
}

Department of Plastic and Reconstructive Surgery, The First Hospital of Jilin University, Changchun, Jilin 130021, P.R. China

Received June 15, 2016; Accepted April 13, 2017

DOI: $10.3892 / \mathrm{mmr} .2017 .6779$

\begin{abstract}
Scarring is the inevitable consequence of wound repair, which may cause significant physical and mental pain to patients when excessive. Bone morphogenetic protein-7 (BMP-7) has been proved to inhibit TGF- $\beta$-induced fibrosis in various tissues including dermal papilla cells. However, the effect of BMP-7 on hypertrophic scarring, a common proliferative disorder of dermal fibroblasts, has not been determined. To overcome this problem, the present study established a mouse model of thermal injury to investigate the inhibitory effects of BMP-7 on scar formation. The histological analysis of scar tissues was performed by H\&E and Masson's trichrome staining. Western blot assay was used to determine the level changes of related proteins and TUNEL assay was performed to assess the apoptosis of scar tissues. The results demonstrated that BMP-7 promoted wound healing and inhibited scar formation when compared with untreated mice. Collagen deposition and the expression of fibrotic proteins were suppressed in the scar tissues of mice treated with BMP-7. In addition, BMP-7 induced fibroblast apoptosis in scar tissues. Furthermore, activation of the BMP-7/Smad1/5/8 signaling pathway may have been involved in the inhibitory effects of BMP-7 on scar formation. In conclusion, the results of the present study indicate that BMP-7 may inhibit excessive scar formation via activation of the BMP-7/Smad1/5/8 signaling pathway. The results present a potential alternative therapeutic strategy for the treatment of hypertrophic scarring.
\end{abstract}

\section{Introduction}

Pathological scarring is one of the most common complications that occurs following severe wounds and burns, and remains an important issue that is difficult to treat by plastic surgery (1). Pathological scars affect an individual's appearance and may

Correspondence to: Dr Duo Zhang, Department of Plastic and Reconstructive Surgery, The First Hospital of Jilin University, 71 Xinmin Avenue, Changchun, Jilin 130021, P.R. China

E-mail: zhangduo_zd@sina.com

Key words: scar formation, bone morphogenetic protein-7, fibrosis, apoptosis, Smad1/5/8 lead to varying degrees of functional impairment, which may have psychological and economic consequences for patients. Currently, the mechanisms underlying the formation of pathological scars are not well established. Current therapeutic methods for the treatment of pathological scars include drug therapy, compression therapy, radiotherapy, laser therapy, biotherapy, gene therapy and surgical treatment; however, the therapeutic efficacy of these methods requires improvement (2).

During the wound-healing process, the proliferation of fibroblasts and deposition of collagen are conducive to wound healing. However, the hyperproliferation of fibroblasts and excessive collagen deposition may lead to hypertrophic scar formation (3). The transforming growth factor (TGF)- $\beta 1$ cytokine is closely associated with scar formation, and overexpression of TGF- $\beta 1$ has been demonstrated to promote scar formation (4). Bone morphogenetic protein-7 (BMP-7) is a member of the TGF- $\beta$ superfamily. Increasing evidence suggests that BMP-7 attenuates TGF- $\beta$-induced fibrogenesis in various tissues (5). Bi et al (6) reported that the TGF- $\beta 1 / \mathrm{BMP}-7$ ratio regulated liver fibrosis in rats. An additional study demonstrated that maintaining BMP-7 levels and inhibiting TGF- $\beta 1$ attenuates the progression of renal fibrosis (7). Furthermore, it was previously demonstrated that BMP-7 inhibits TGF- $\beta 1$-induced fibroblast-like differentiation of rat dermal papilla cells (8). Based on these reports, the authors of the current study hypothesized that BMP-7 may serve a role in promoting wound healing and attenuating scar formation.

In the present study, a mouse model of thermal injury was established. The effect of exogenous BMP-7 treatment on scar formation and its potential mechanisms were then investigated, and the results present a novel theoretical basis for the treatment of skin scars.

\section{Materials and methods}

Chemicals and antibodies. Recombinant human BMP-7 was purchased from Cusabio Biotech Co., Ltd (Wuhan, China) and dissolved in sterile saline prior to use. The primary antibodies against the following proteins employed were as follows: Collagen I (BA0323; Wuhan Boster Biological Technology, Ltd., Wuhan, China); collagen III (BM1625; Wuhan Boster Biological Technology, Ltd.); $\alpha$-smooth muscle actin ( $\alpha$-SMA; BM0002; Wuhan Boster Biological Technology, Ltd.); connective tissue growth factor (CTGF; ab6992; Abcam, Cambridge, 
UK); TGF-ק1 (sc-146; Santa Cruz Biotechnology, Inc., Dallas, TX, USA); B-cell lymphoma 2 (BCL2; BA0412; Wuhan Boster Biological Technology, Ltd.); BCL2-associated X (Bax; BA0315; Wuhan Boster Biological Technology, Ltd.); cleaved caspase-3 (ab2302; Abcam); Smad1/5/8 (sc-6031R; Santa Cruz Biotechnology, Inc.); phosphorylated (p)-Smad1/5/8 (sc-12353; Santa Cruz Biotechnology, Inc.); and $\beta$-actin (sc-47778; Santa Cruz Biotechnology, Inc.). The secondary antibodies were HRP-conjugated Goat anti-Rabbit IgG (WLA023; Wanleibio, Shenyang, China) and HRP-conjugated Goat anti-Mouse IgG (WLA024; Wanleibio, Shenyang, China).

Animals and experimental protocol. C57BL/6 mice were purchased from Vital River Laboratories Co., Ltd. (Beijing, China). The mice (6 mice/cage) were reared at $22 \pm 1^{\circ} \mathrm{C}$ and $45-55 \%$ humidity, fed standard laboratory chow and had free access to food and water throughout the experiment. A total of 30 male C57BL/6 mice (18-20 g, 8 weeks) were randomly divided into the following five groups ( $\mathrm{n}=6 / \mathrm{group}$ ): Control, thermal injury, thermal injury + BMP-7 $(25 \mu \mathrm{g})$, thermal injury + BMP-7 $(50 \mu \mathrm{g})$, and thermal injury + BMP-7 $(100 \mu \mathrm{g})$. The mouse model of thermal injury was established according to Walker's method with some modifications (9). Briefly, the mice were anesthetized by intraperitoneal injection of $10 \%$ chloral hydrate $(3.5 \mathrm{ml} / \mathrm{kg})$. The hair on the back was then removed to expose the dorsal skin. Following disinfection, a $2-\mathrm{cm}$ diameter plastic pipe was placed directly onto the dorsal skin, with one end was in close contact with the skin and the other was injected with boiling water. The boiling water remained in contact with the skin for $10 \mathrm{sec}$. To prevent infection, the mice that received thermal injury were housed individually, and the mice in the thermal injury + BMP-7 $(25 \mu \mathrm{g})$, thermal injury + BMP-7 $(50 \mu \mathrm{g})$ and thermal injury + BMP-7 $(100 \mu \mathrm{g})$ groups were subcutaneously injected once with BMP-7 at the respective dosages immediately after the treatment with boiling water. Mice in the control and thermal injury groups were subcutaneously injected with an equal volume of sterile saline. All mice were euthanized at 15 days following thermal injury, and scalded skin tissues were obtained for subsequent experiments. All animal experiments were performed in strict accordance with international ethical guidelines (10) and the National Institutes of Health Guide concerning the Care and Use of Laboratory Animals (11). All animal experiments were approved by the Institutional Animal Care and Use Committee of Jilin University (Changchun, China).

Wound healing observation. At 0, 3, 7, 10 and 14 days following thermal injury, the healing of scalded skin in mice from all experimental groups was observed and photographed. The wound area was measured using Photoshop CS5 software (Adobe Systems, Inc., Beijing, China).

Histological analysis. The scar tissues and normal skin tissues were collected at 15 days following thermal injury, and were fixed in $4 \%$ paraformaldehyde for $24 \mathrm{~h}$ at room temperature. Tissues were washed in an ethanol series before they were embedded in paraffin and sectioned at 5- $\mu \mathrm{m}$ thickness. Subsequently, routine hematoxylin and eosin (H\&E) and Masson's trichrome staining were performed according to standard procedures (12). Morphological alterations in scar tissues were determined by $\mathrm{H} \& \mathrm{E}$ staining, and scar tissue fibrosis was evaluated by Masson's trichrome staining. Representative images were observed and photographed under a light microscope at x200 magnification.

Western blotting. The protein expression levels in scar tissues were determined by western blot analysis. Briefly, total protein in tissues from different groups was extracted using a lysis buffer (Beyotime Institute of Biotechnology, Haimen, China). Following centrifugation at $10,000 \mathrm{x} \mathrm{g}$ and $4^{\circ} \mathrm{C}$ for $10 \mathrm{~min}$, the concentration of protein was determined using a BCA kit (Beyotime Institute of Biotechnology).Equal quantities of protein $(40 \mu \mathrm{g})$ were subsequently separated by 8,10 or $13 \%$ SDS-PAGE and transferred to polyvinylidene difluoride membranes (EMD Millipore, Billerica, MA, USA). The membranes were then blocked with 5\% skimmed milk for $1 \mathrm{~h}$ at room temperature, before they were incubated with anti-collagen I (1:400), anti-collagen III (1:400), anti- $\alpha$-SMA (1:400), anti-CTGF $(1: 1,000)$, anti-TGF- $\beta 1$ (1:200), anti-BCL2 (1:400), anti-Bax (1:400), anti-cleaved caspase-3 (1:1,000), anti-Smad1/5/8 (1:200), anti-p-Smad1/5/8 (1:200) and anti- $\beta$-actin $(1: 1,000)$ primary antibodies at $4{ }^{\circ} \mathrm{C}$ overnight. Membranes were subsequently incubated with goat anti-rabbit/mouse secondary antibodies $(1: 5,000)$ for $45 \mathrm{~min}$ at $37^{\circ} \mathrm{C}$. The bands were visualized using an enhanced chemiluminescence detection reagent (Beyotime Institute of Biotechnology). The bands were photographed and the optical densities were quantified using Gel-Pro Analyzer software version 4.5 (Media Cybernetics, Bethesda, USA).

TUNEL staining. The level of apoptosis in scar tissues was assessed by TUNEL staining using an In Situ Cell Death Detection kit (Roche; Sigma-Aldrich; Merck KGaA, Darmstadt, Germany) according to the manufacturer's instructions. Briefly, scar tissues were washed in three times in phosphate-buffered saline and fixed in $4 \%$ paraformaldehyde for $12 \mathrm{~h}$ at room temperature. Tissues were subsequently permeabilized by incubating with $0.1 \%$ Triton X-100 for $8 \mathrm{~min}$ at room temperature. Slides were then incubated with $50 \mu 1$ TUNEL reaction mixture (enzyme solution:label solution=1:9) for $60 \mathrm{~min}$ at $37^{\circ} \mathrm{C}$ in the dark. Following washing with PBS for $5 \mathrm{~min}$ three times, the slides were stained with $50 \mu 1$ 3'3 diaminobenzidine (DA1010; Beijing Solarbio Science \& Technology Co., Ltd., Beijing, China) at room temperature and immediately placed in water just as it became dark. Then the slides were washed for $5 \mathrm{~min}$ three times, observed and imaged at $x 400$ magnification under a light microscope, and the number of apoptotic cells was determined.

Statistical analysis. GraphPad Prism software (version, 5.0; GraphPad Software, Inc., La Jolla, CA, USA) was used for statistical analyses. All data are presented as the mean + standard deviation. One-way analysis of variance followed by Bonferroni's post hoc test was performed to compare the results among groups. $\mathrm{P}<0.05$ was considered to indicate a statistically significant difference.

\section{Results}

BMP-7 inhibits scar formation in a mouse model of thermal injury. The mouse model of thermal injury was first established 


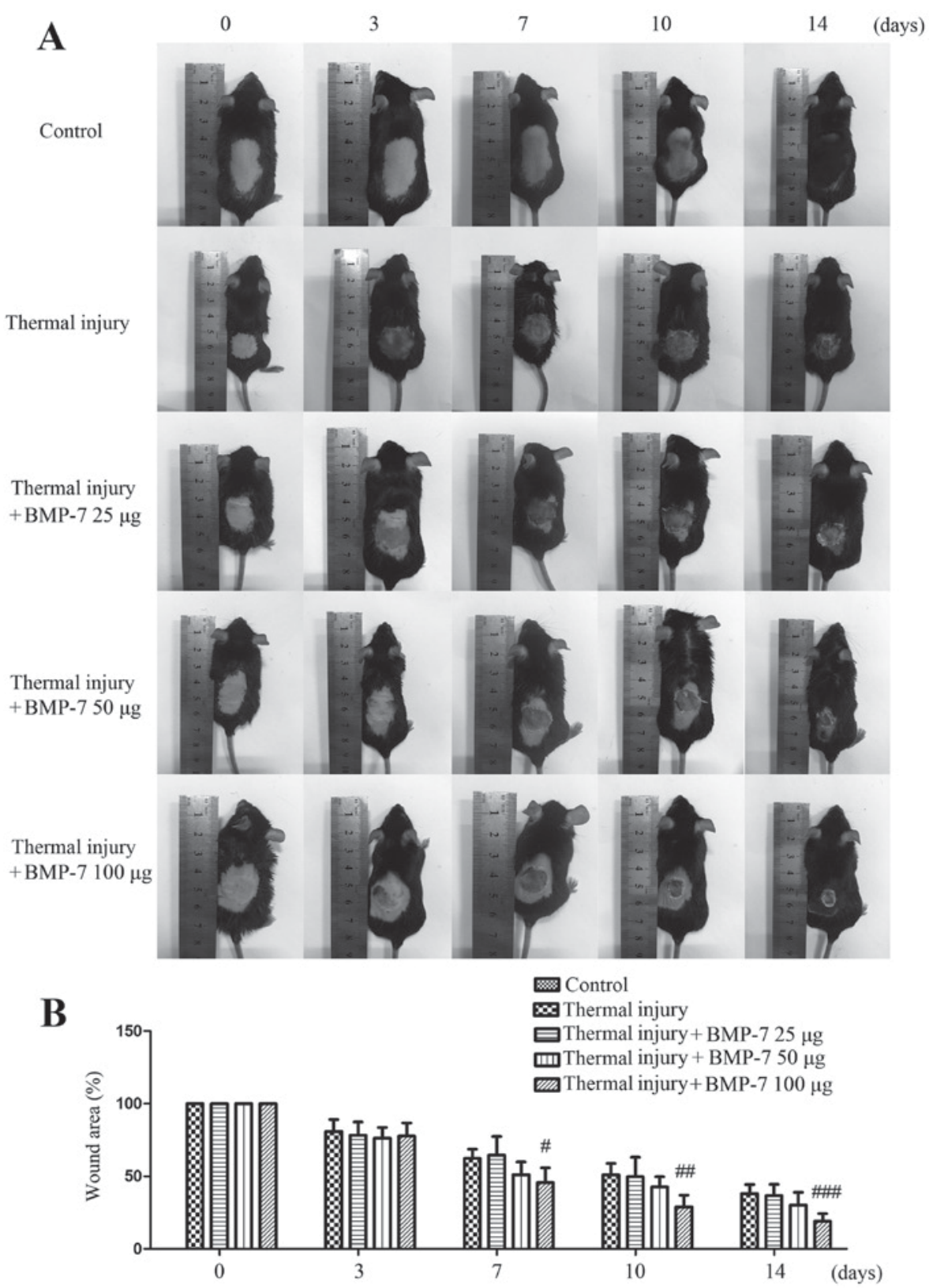

Figure 1. BMP-7 facilitates wound healing and prevents scar formation in a mouse model of thermal injury. (A) The model was first established and mice were subcutaneously injected with saline or 25,50 or $100 \mu \mathrm{g}$ BMP-7. At 0, 3, 7, 10 and 14 days following thermal injury, photos of mice from each group were obtained and the representative images are presented. (B) The average wound area of mice in each group was calculated, and the percentage wound area relative to the area at day 0 is presented. The results are presented as the mean + standard deviation $(n=6)$, and are representative of three independent experiments. ${ }^{\#} \mathrm{P}<0.05,{ }^{\# \#} \mathrm{P}<0.01$ and ${ }^{\# \# \#} \mathrm{P}<0.001$ vs. untreated thermal injury group. BMP-7, bone morphogenetic protein-7.

in order to evaluate the effect of BMP-7 on scar formation. As demonstrated in Fig. 1, the scalded wound surface of mice in each experimental group was examined. Among the three different doses of BMP-7 tested, $100 \mu \mathrm{g}$ significantly decreased wound size and inhibited scar formation at 7, 10 and 14 days following thermal injury when compared with the untreated thermal injury group.

H\&E staining was used to examine histopathological morphologic alterations in the scalded tissues. The results demonstrated that the epidermis and dermis in the scalded skin tissue were thicker when compared with the control group (Fig. 2). In addition, there was obvious inflammatory cell infiltration, and the skin suffered structural damage to a certain degree. However, treatment with BMP-7 attenuated these histopathological morphologic alterations when compared with tissues from the untreated thermal injury group (Fig. 2). These results indicate that BMP-7 demonstrates a potential inhibitory effect on scar formation.

BMP-7 suppresses collagen deposition and fibrotic protein expression in scar tissues. Collagen deposition in the scar tissues of mice from each experimental group was then determined. Masson's trichrome staining was performed to evaluate collagen fiber area density and arrangement in scar tissues. As demonstrated in Fig. 3A, the collagen fibers were stained blue. BMP-7 treatment was associated with a reduced collagen area in scar tissues when compared with the untreated thermal injury group. The arrangement of collagen fibers in the scar tissues 


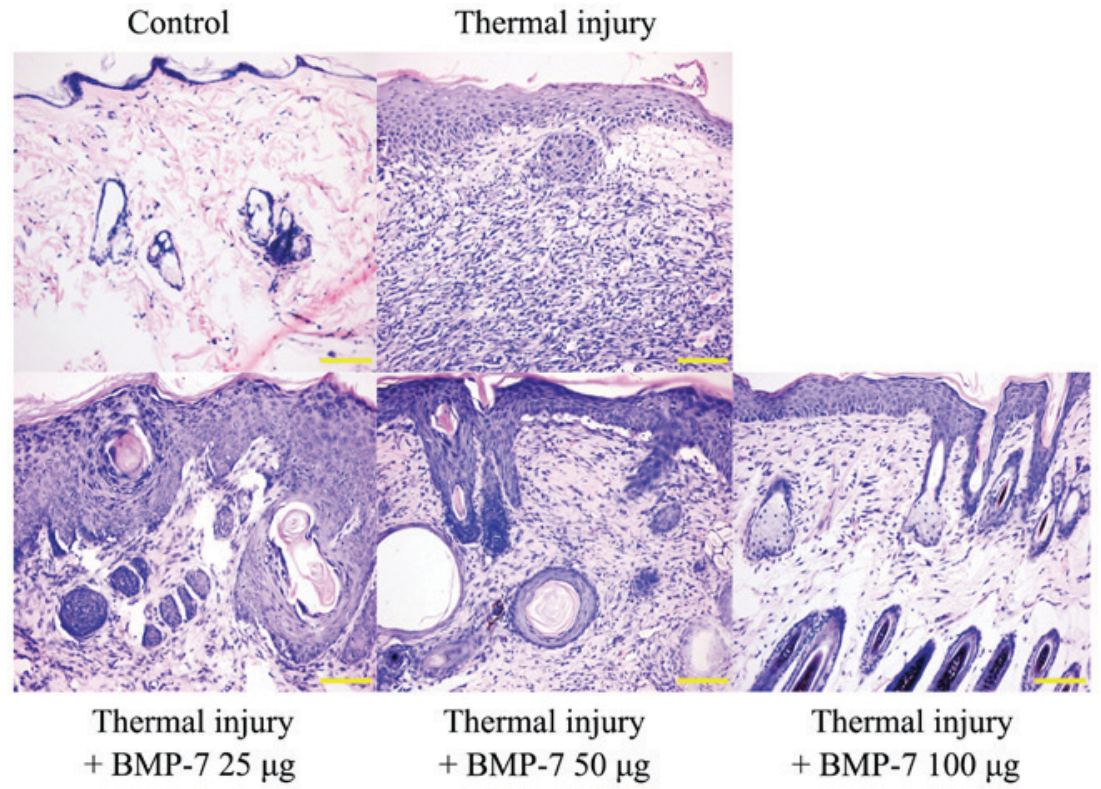

Figure 2. BMP-7 inhibits histopathological morphologic alterations in scald tissues. Histomorphological alterations in scar tissues were observed by hematoxylin and eosin staining at 15 days following thermal injury (red arrows, epidermis; yellow arrows, dermis; magnification, x200; scale bars, $100 \mu \mathrm{m}$ ). BMP-7, bone morphogenetic protein-7.

A

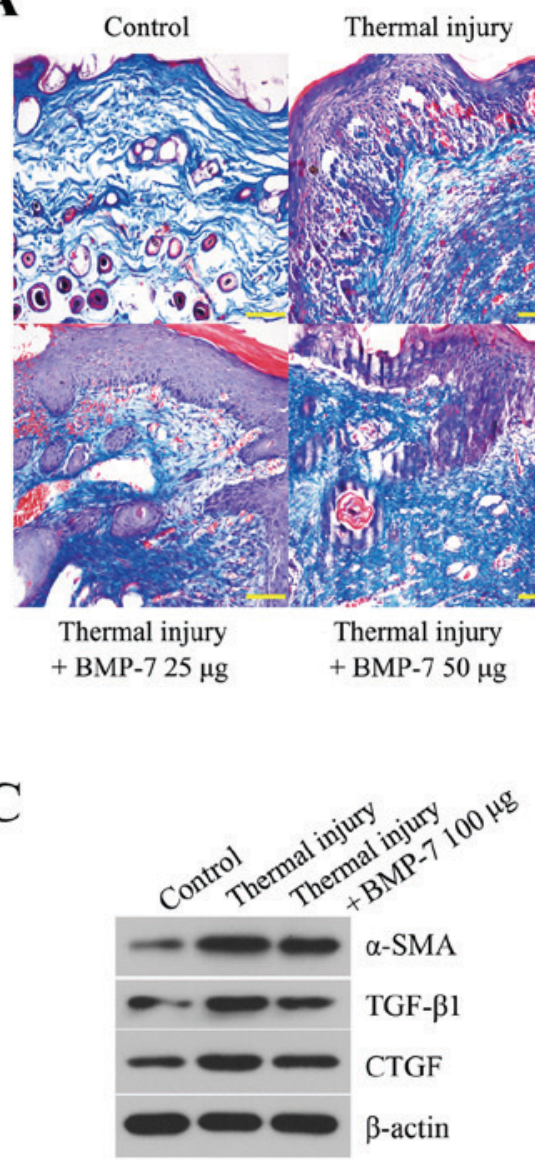

B
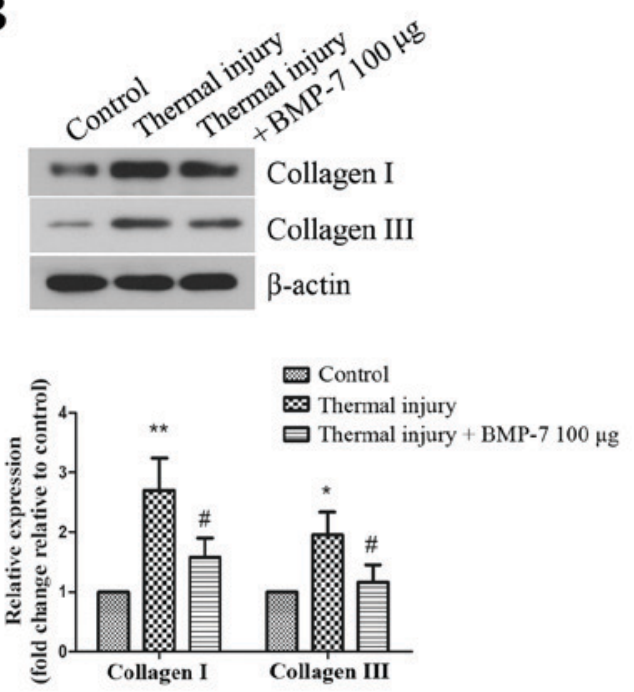

Figure 3. BMP-7 suppresses collagen deposition and fibrotic protein expression in scar tissues. (A) Masson's trichrome staining was performed to examine collagen fibers in skin tissues of mice from the different groups (magnification, x200; scale bars, $100 \mu \mathrm{m}$ ). (B) The protein expression levels of collagen I and collagen III were determined by western blot analysis, and protein levels were quantified by densitometric analysis. $\beta$-actin was used as a loading control. (C) Protein expression levels of fibrosis-associated proteins, $\alpha$-SMA, TGF- $\beta 1$ and CTGF, as determined by western blot analysis. Protein levels were quantified by densitometric analysis. The results are presented as the mean + standard deviation $(\mathrm{n}=6)$, and are representative of three independent experiments. ${ }^{*} \mathrm{P}<0.05$ and ${ }^{* *} \mathrm{P}<0.01$ vs. control group; ${ }^{*} \mathrm{P}<0.05$ vs. untreated thermal injury group. BMP-7, bone morphogenetic protein-7; $\alpha$-SMA, $\alpha$-smooth muscle actin; TGF, transforming growth factor; CTGF, connective tissue growth factor. 
of mice from all BMP-7-treated groups was more loose and regular when compared with mice in the untreated thermal injury group.

Based on the above results, BMP-7 with the dose of $100 \mu \mathrm{g}$ had the most inhibitory effect on scarring. Therefore, $100 \mu \mathrm{g}$ BMP-7 was chosen for the following experiments. The protein expression levels of collagen I and collagen III were detected by western blot analysis. As demonstrated in Fig. 3B, the levels of collagen I and III were increased significantly in the thermal injury group when compared with the control group. By contrast, treatment with $100 \mu \mathrm{g}$ BMP-7 significantly reduced collagen I and III protein expression levels when compared with the thermal injury group.

$\alpha$-SMA, TGF- $\beta 1$ and CTGF are proteins that are involved in promoting fibrosis, which leads to scar formation (13). In the present study, the expression levels of these proteins in mice from all experimental groups were determined by western blot analysis. As demonstrated in Fig. 3C, thermal injury induced a significant increase in the protein expression levels of $\alpha$-SMA, TGF- $\beta 1$ and CTGF when compared with the control group. By contrast, treatment with $100 \mu \mathrm{g}$ BMP-7 significantly reduced the protein expression levels of $\alpha$-SMA, TGF- $\beta 1$ and CTGF when compared with the mice in the untreated thermal injury group.

BMP-7 induces fibroblast apoptosis in scar tissues. The level of apoptosis in scar tissues was determined using a TUNEL assay. As demonstrated in Fig. 4A, the level of TUNEL-positive staining was lower in the scar tissues of mice in the thermal injury group compared with those of the control group. By contrast, treatment with $100 \mu \mathrm{g}$ BMP-7 markedly enhanced the level of apoptosis in scar tissues when compared with the thermal injury group (Fig. 4A). In order to validate these observations, the expression of apoptosis-associated proteins was then determined by western blot analysis. As demonstrated in Fig. 4B, treatment with $100 \mu \mathrm{g}$ BMP-7 significantly decreased the protein expression levels of BCL2, and significantly increased the levels of Bax and cleaved caspase-3 when compared with the thermal injury group.

BMP-7 activates the BMP-7/Smad1/5/8 signaling pathway. In order to investigate the molecular mechanisms underlying BMP-7-mediated suppression of scar formation, the present study examined the BMP-7/Smad1/5/8 signaling pathway. As demonstrated in Fig. 5, the protein expression levels of BMP-7 and $\mathrm{p}-\mathrm{Smad} 1 / 5 / 8$ were significantly decreased in scar tissues induced by thermal injury when compared with the control group. However, injection of exogenous $100 \mu \mathrm{g}$ BMP-7 significantly enhanced the level of $\mathrm{p}$-Smad1/5/8 when compared with the untreated thermal injury group.

\section{Discussion}

To the best of the author's knowledge, the present study is the first to report that BMP-7 treatment suppresses scar formation in a mouse model of thermal injury. Pathological scarring is a common fibrotic disease that occurs during wound healing (14). Currently, there are numerous treatments with clearly proven efficiency or doubtful benefits for this condition. However, the optimal treatment has yet to be identified. Therefore, investigating novel therapeutic methods and understanding the underlying mechanisms is important for improving treatment strategies for patients with pathological scarring. BMP-7 has been reported to inhibit TGF- $\beta 1$-induced epithelial-mesenchymal transition, and has therefore been identified as an anti-fibrosis cytokine with potential applications in the treatment of fibrotic diseases (15). Until recently, the effect of BMP-7 on scar formation was unknown. In the present study, recombinant human BMP-7 was injected into the scalded skin of mice, and the effect on scar formation and the underlying mechanisms were investigated. The results demonstrated that exogenous BMP-7 promoted wound healing and inhibited scar formation. This provides a novel insight into the molecular role of BMP-7 in skin wound healing, and indicates that BMP-7 may present a potential therapeutic target for the treatment of pathological scarring.

It is generally accepted that hypertrophic scars are primarily characterized by disturbances in collagen metabolism. Collagen, as the major component of extracellular matrix, is the structural and functional protein of skin. However, upon serious trauma, collagen deposits in hypertrophic scar fibroblasts and the expression of collagen I and III are increased (16). The results of the present study demonstrated that BMP-7 treatment reduced excessive collagen deposition in the scar tissues induced by thermal injury, and the thermal injury-induced increases in the expression of collagen I and III were significantly inhibited by BMP-7 treatment. The cytoskeletal protein, $\alpha$-SMA, is a typical marker of a contractile phenotype in myofibroblasts (17). The skin exhibits excessive contraction during wound healing as a result of $\alpha$-SMA overexpression at the site of injury, which finally induces scar formation $(18,19)$. Therefore, $\alpha$-SMA is considered to be closely associated with scar contracture, and inhibiting the production of $\alpha$-SMA is conducive to preventing scarring during wound repair. According to the results of the current study, the protein expression levels of $\alpha$-SMA were upregulated in thermal injury-induced scar tissues, which was significantly inhibited by BMP-7 treatment. Skin fibroblasts synthesize and secrete CTGF, which demonstrates a strong regulatory effect on the proliferation and differentiation of fibroblasts (20). As abnormally high expression of CTGF is known to serve a key role in the development of scar formation, CTGF has become a focus of anti-scarring research (21). In addition, CTGF is a direct downstream effector of TGF- $\beta 1$, and mediates the profibrogenic effects of TGF- $\beta 1$ in various diseases, such as cicatrix, which is characterized by the scarring of healed wounds (22-24). The results of the present study demonstrated that BMP-7 significantly inhibited the expression of CTGF in thermal injury-induced wound tissues. Taking into account the results of previous studies, the anti-fibrotic effects of BMP-7 were significant, and may have therefore been the primary mechanism for inhibiting scar formation.

Apoptosis is a programmed cell death mechanism. A previous study has demonstrated that the level of apoptosis in pathological scar-derived fibroblasts is lower when compared with normal skin (25). An additional report indicated that the imbalance between fibroblast proliferation and apoptosis is an important factor in scar formation (26). Therefore, inducing apoptosis in fibroblasts may be an effective strategy for the treatment of pathological scarring. The results of the present study demonstrated that the level of apoptosis in scar tissues 
A

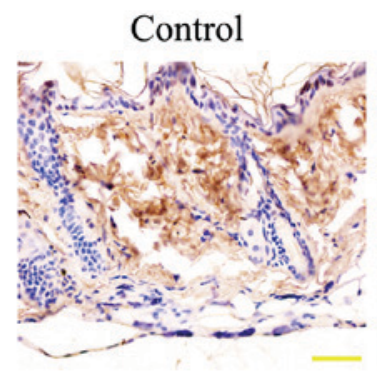

Thermal injury

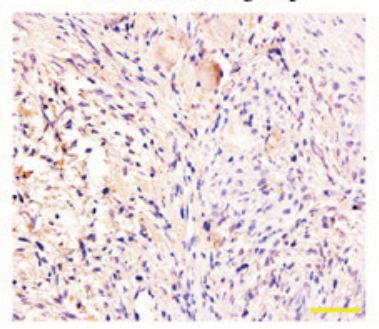

Thermal injury

+ BMP-7 $100 \mu \mathrm{g}$

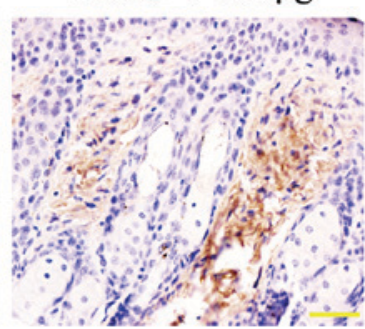

B

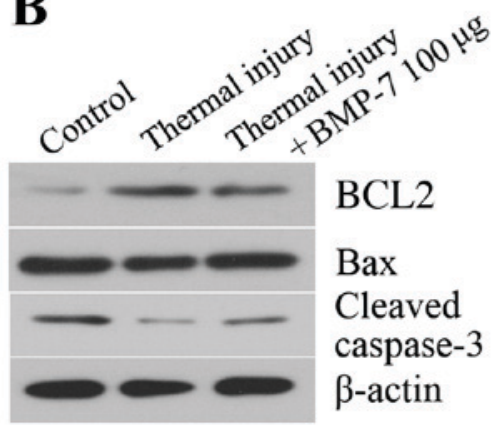

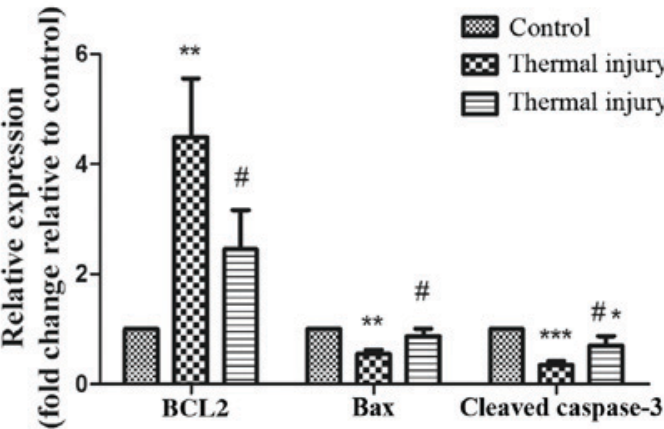

Figure 4. BMP-7 induces fibroblast apoptosis in scar tissues. (A) Effect of BMP-7 on apoptosis in scar tissues was evaluated using a TUNEL assay (magnification, $\mathrm{x} 400$; scale bars, $50 \mu \mathrm{m}$ ). (B) The protein expression levels of apoptosis-associated proteins, BCL2, Bax and cleaved caspase-3 were assessed by western blot analysis, and protein levels were quantified by densitometric analysis. $\beta$-actin was used as a loading control. The results are presented as the mean + standard deviation $(\mathrm{n}=6)$, and are representative of three independent experiments. ${ }^{*} \mathrm{P}<0.05,{ }^{* *} \mathrm{P}<0.01$ and ${ }^{* * *} \mathrm{P}<0.001$ vs. control group; ${ }^{\#} \mathrm{P}<0.05$ vs. untreated thermal injury group. BMP-7, bone morphogenetic protein-7; BCL2, B-cell lymphoma 2; Bax, BCL2-associated X.

A

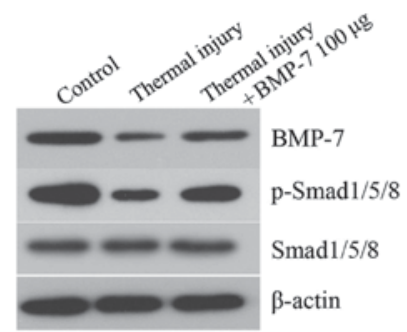

B

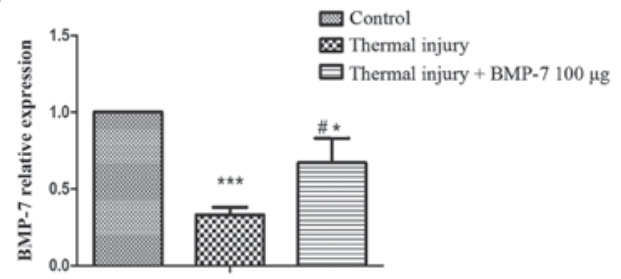

C

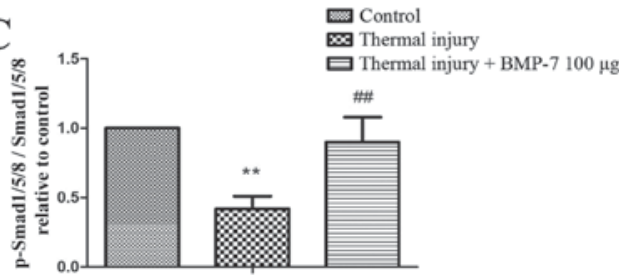

Figure 5. BMP-7 promotes activation of the BMP-7/Smad1/5/8 signaling pathway. (A) The protein expression levels of BMP-7, p-Smad1/5/8 and Smad1/5/8 were detected by western blot analysis. $\beta$-actin was used as a loading control. Results of densitometric analysis for (B) BMP-7 and (C) $\mathrm{p}$-Smad1/5/8/Smad1/5/8 protein expression. The results are presented as the mean + standard deviation $(n=6)$, and are representative of three independent experiments. ${ }^{*} \mathrm{P}<0.05,{ }^{* *} \mathrm{P}<0.01$ and ${ }^{* * *} \mathrm{P}<0.001$ vs. control group; ${ }^{*} \mathrm{P}<0.05$ and ${ }^{\# \#} \mathrm{P}<0.01$ vs. untreated thermal injury group. BMP-7, bone morphogenetic protein-7; p-, phosphorylated-. was increased significantly following treatment with BMP-7 when compared with untreated controls. The BCL2 protein family regulates cell apoptosis, and involves two types of functionally opposing proteins: Anti-apoptotic proteins, such as BCL2, and pro-apoptotic proteins, such as Bax. The BCL2/Bax ratio is a major factor for the regulation of apoptosis. Caspase- 3 is an important member of the caspase cascade reaction, and functions as a key downstream regulatory protein in apoptotic signaling pathways. Activation of caspase-3 is responsible for the induction of the majority of apoptotic signaling pathways (27). The results of the current study demonstrated that BMP-7 treatment significantly reduced the BCL2/Bax ratio and increased the expression of cleaved caspase- 3 when compared with the untreated thermal injury group. In addition, the results indicated that the level of apoptosis in scar tissues from mice in the thermal injury group were reduced when compared with the control group, which may have been due to the hyperproliferation of fibroblasts in scar tissues. However, the molecular mechanisms underlying this reduction in apoptosis in scar tissues requires further investigation.

The Smad signaling pathway serves important roles in collagen metabolism, and has been confirmed as one of the most extensively studied signaling pathways in scar formation. Among different Smad proteins, p-Smad1/5/8 is used as a marker of BMP/Smad signaling (28). BMP-7 serves a role in preventing fibrosis by activating the $\mathrm{BMP} / \mathrm{Smad}$ signaling pathway in various diseases. Yang et al (28) demonstrated that BMP-7 inhibited silica-induced pulmonary fibrosis in rats by upregulating the expression of $\mathrm{p}$-Smad1/5/8. In addition, $\mathrm{p}-\mathrm{Smad} 1 / 5 / 8$ was demonstrated to be involved in BMP-7 
signaling pathway-induced anti-fibrotic effects on fibrous capsule formation on the surface of orthopedic metallic prosthetic implants (29). Furthermore, increased expression of p-Smad1/5/8 was observed in mediating the anti-fibrosis effects of BMP-7 in the cornea (30) and lung (31). Consistent with the results of these studies, the present study demonstrated that BMP-7 treatment significantly increased the expression of $\mathrm{p}$-Smad1/5/8 at thermal injury sites when compared with untreated controls.

In conclusion, the present study provides evidence that BMP-7 promotes wound healing, inhibits collagen formation and the progression of fibrosis, and induces fibroblast apoptosis. Activation of the BMP/Smad signaling pathway may be the underlying mechanism responsible for the suppressive effects of BMP-7 on scar formation. Although the effect of BMP-7 on scar formation and its potential mechanisms require further research, the authors suggest that BMP-7 may be a potential therapeutic target for the treatment of patients with pathological scarring.

\section{References}

1. Sun X, Cheng L, Zhu W, Hu C, Jin R, Sun B, Shi Y, Zhang Y and Cui W: Use of ginsenoside Rg3-loaded electrospun PLGA fibrous membranes as wound cover induces healing and inhibits hypertrophic scar formation of the skin. Colloids Surf B Biointerfaces 115: 61-70, 2014.

2. Tredget EE, Levi B and Donelan MB: Biology and principles of scar management and burn reconstruction. Surg Clin North Am 94: 793-815, 2014.

3. Zeng G, Zhong F, Li J, Luo S and Zhang P: Resveratrol-mediated reduction of collagen by inhibiting proliferation and producing apoptosis in human hypertrophic scar fibroblasts. Biosci Biotechnol Biochem 77: 2389-2396, 2013.

4. Jagadeesan $J$ and Bayat A: Transforming growth factor beta (TGFbeta) and keloid disease. Int J Surg 5: 278-285, 2007.

5. Weiskirchen R and Meurer SK: BMP-7 counteracting TGF-beta1 activities in organ fibrosis. Front Biosci (Landmark Ed) 18 1407-1434, 2013

6. Bi WR, Xu GT, Lv LX and Yang CQ: The ratio of transforming growth factor- $\beta 1 /$ bone morphogenetic protein- 7 in the progression of the epithelial-mesenchymal transition contributes to rat liver fibrosis. Genet Mol Res 13: 1005-1014, 2014.

7. Tasanarong A, Kongkham S, Thitiarchakul S and Eiam-Ong S: Vitamin E ameliorates renal fibrosis in ureteral obstruction: Role of maintaining BMP-7 during epithelial-to-mesenchymal transition. J Med Assoc Thai 94 (Suppl 7): S10-S18, 2011.

8. Bin S, Li HD, Xu YB, Qi SH, Li TZ, Liu XS, Tang JM and Xie JL: BMP-7 attenuates TGF- $\beta 1$-induced fibroblast-like differentiation of rat dermal papilla cells. Wound Repair Regen 21: 275-281, 2013.

9. Walker HL and Mason AD Jr: A standard animal burn. J Trauma 8: 1049-1051, 1968

10. Tian X, Zheng Y, Li Y, Shen Z, Tao L, Dou X, Qian J and Shen H: Psychological stress induced zinc accumulation and up-regulation of ZIP14 and metallothionein in rat liver. BMC Gastroenterol 14: 32, 2014.

11. Zhang M, Sun G, Shen A, Liu L, Ding J and Peng J: Patrinia scabiosaefolia inhibits the proliferation of colorectal cancer in vitro and in vivo via G1/S cell cycle arrest. Oncol Rep 33: 856-860, 2015.

12. Apgar JM, Juarranz A, Espada J, Villanueva A, Cañete M and Stockert JC: Fluorescence microscopy of rat embryo sections stained with haematoxylin-eosin and Masson's trichrome method. J Microsc 191: 20-27, 1998
13. Mun JH, Kim YM, Kim BS, Kim JH, Kim MB and Ko HC: Simvastatin inhibits transforming growth factor- $\beta 1$-induced expression of type I collagen, CTGF and $\alpha$-SMA in keloid fibroblasts. Wound Repair Regen 22: 125-133, 2014.

14. Kopecki Z and Cowin AJ: Flightless I: An actin-remodelling protein and an important negative regulator of wound repair. Int J Biochem Cell Biol 40: 1415-1419, 2008.

15. Weiskirchen R, Meurer SK, Gressner OA, Herrmann J, Borkham-Kamphorst E and Gressner AM: BMP-7 as antagonist of organ fibrosis. Front Biosci (Landmark Ed) 14: 4992-5012, 2009.

16. Ghahary A, Shen YJ, Scott PG, Gong Y and Tredget EE: Enhanced expression of mRNA for transforming growth factor-beta, type I and type III procollagen in human post-burn hypertrophic scar tissues. J Lab Clin Med 122: 465-473, 1993.

17. Muchaneta-Kubara EC and el Nahas AM: Myofibroblast phenotypes expression in experimental renal scarring. Nephrol Dial Transplant 12: 904-915, 1997.

18. Agarwal C, Britton ZT, Alaseirlis DA, Li Y and Wang JH: Healing and normal fibroblasts exhibit differential proliferation, collagen production, alpha-SMA expression, and contraction. Ann Biomed Eng 34: 653-659, 2006.

19. Nedelec B, Ghahary A, Scott PG and Tredget EE: Control of wound contraction. Basic and clinical features. Hand Clin 16: 289-302, 2000.

20. Oemar BS and Lüscher TF: Connective tissue growth factor. Friend or foe? Arterioscler Thromb Vasc Biol 17: 1483-1489, 1997.

21. Grotendorst GR, Okochi $\mathrm{H}$ and Hayashi N: A novel transforming growth factor beta response element controls the expression of the connective tissue growth factor gene. Cell Growth Differ 7: 469-480, 1996

22. Bradham DM, Igarashi A, Potter RL and Grotendorst GR: Connective tissue growth factor: A cysteine-rich mitogen secreted by human vascular endothelial cells is related to the SRC-induced immediate early gene product CEF-10. J Cell Biol 114: 1285-1294, 1991.

23. Yatomi M, Hisada T, Ishizuka T, Koga Y, Ono A, Kamide Y, Seki K, Aoki-Saito H, Tsurumaki H, Sunaga N, et al: 17(R)-resolvin D1 ameliorates bleomycin-induced pulmonary fibrosis in mice. Physiol Rep 3: pii: e12628, 2015.

24. Song R, Li G and Li S: Aspidin PB, a novel natural anti-fibrotic compound, inhibited fibrogenesis in TGF- $\beta 1$-stimulated keloid fibroblasts via PI-3K/Akt and Smad signaling pathways. Chem Biol Interact 238: 66-73, 2015.

25. Wang H, Chen Z, Li XJ, Ma L and Tang YL: Anti-inflammatory cytokine TSG-6 inhibits hypertrophic scar formation in a rabbit ear model. Eur J Pharmacol 751: 42-49, 2015.

26. Akasaka Y, Fujita K, Ishikawa Y, Asuwa N, Inuzuka K, Ishihara M, Ito M, Masuda T, Akishima Y, Zhang L, et al: Detection of apoptosis in keloids and a comparative study on apoptosis between keloids, hypertrophic scars, normal healed flat scars, and dermatofibroma. Wound Repair Regen 9: 501-506, 2001.

27. Liu Q, Si T, Xu X, Liang F, Wang L and Pan S: Electromagnetic radiation at $900 \mathrm{MHz}$ induces sperm apoptosis through bcl-2, bax and caspase-3 signaling pathways in rats. Reprod Health 12: $65,2015$.

28. Yang G, Zhu Z, Wang Y, Gao A, Niu P and Tian L: Bone morphogenetic protein-7 inhibits silica-induced pulmonary fibrosis in rats. Toxicol Lett 220: 103-108, 2013.

29. Tan HC, Poh CK, Cai Y and Wang W: Anti-fibrosis effect of BMP-7 peptide functionalization on cobalt chromium alloy. J Orthop Res 31: 983-990, 2013.

30. Tandon A, Sharma A, Rodier JT, Klibanov AM, Rieger FG and Mohan RR: BMP7 gene transfer via gold nanoparticles into stroma inhibits corneal fibrosis in vivo. PLoS One 8: e66434, 2013.

31. Murray LA, Hackett TL, Warner SM, Shaheen F, Argentieri RL, Dudas P, Farrell FX and Knight DA: BMP-7 does not protect against bleomycin-induced lung or skin fibrosis. PLoS One 3: e4039, 2008. 